\section{Hospital Anxiety and Depression Scale}

Helga Peter ${ }^{1}$ und Thomas Penzel ${ }^{2}$

${ }^{1}$ Marburg, Deutschland

${ }^{2}$ Interdisziplinäres Schlafmedizinisches Zentrum, Charité Universitätsmedizin Berlin, Berlin, Deutschland

\section{Definition}

Angst- und Depressionsskala für stationäre Patienten zur Selbstbeurteilung.

Siehe dazu \, „Leistungs-, Schläfrigkeits- und Vigilanzmessung".

\title{
Synonyme
}

HADS 